\title{
Identifying Vehicle Model Parameters Using Remote Mounted Motion Sensor
}

\author{
Y. Hwang, Y. Jeong, I.Kweon, Senior Member, IEEE and S.B.Choi, Member, IEEE
}

\begin{abstract}
The recent developments on advanced driver assistance system(ADAS) have extended the capability of sensor systems from surrounding perception to motion estimation. The motion estimation provides tri-axial velocity and pose measurements, which open potential benefits for control and state estimation through sensor fusion with the vehicle dynamics model. In this paper we propose an identification method for the vehicle single track model parameters including the relative distance between the vehicle center of gravity and the motion sensor. A linearized tire force model and simplified single track vehicle model are constructed with the corresponding sensor kinematics model. We demonstrate the efficacy of identification performance of the proposed method and confirm the feasibility of the usage of ADAS sensor in vehicle dynamics and vice versa.
\end{abstract}

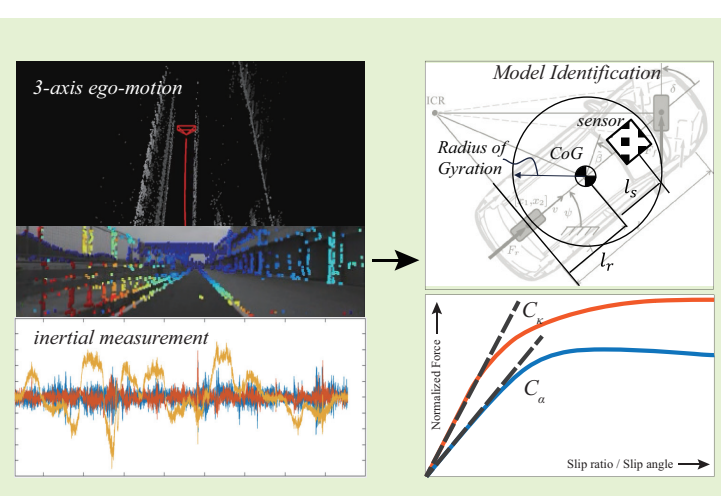

Index Terms - Inertial navigation, Motion estimation, Sensor fusion, System identification, Vehicle dynamics

\section{INTRODUCTION}

$\mathbf{R}$ ESEARCHES on the advanced driver assistance system(ADAS) and self-driving cars have been gaining momentum under the emerging attention of the automotive industry and academia. Over the last decade, numerous massproduced vehicles are produced with state-of-the-art sensor systems, such as LiDAR, RADAR, camera, or real-time kinematics(RTK) GNSS [1]. This innovation of the sensor systems not only offers the advantage of recognizing the surrounding driving scenes but the capability of potential enhancement on the vehicle model and state estimation for the active safety control on various conditions.

The sensor systems for ADAS provide the relative motions of moving objects by detecting distinguishable features from the given scenes and estimate the ego-motion of the vehicle. In order to accomplish precise estimation of the ego-motion, the global navigation satellite system(GNSS) is one of the most attractive options, since it delivers the absolute position and

This work was supported by a grant (20TLRP-C152478-02) from the Transportation Logistics Research Program funded by the Ministry of Land, Infrastructure and Transport(MOLIT) of the Korean government and the Korea Agency for Infrastructure Technology Advancement(KAIA)

Y. Hwang is with the Department of Mechanical Engineering, Korea Advanced Institute of Science and Technology(KAIST), Daejeon 34141 Republic of Korea (e-mail: yoonjinh@kaist.ac.kr).

Y. Jeong is with the Robotics Program, Korea Advanced Institute of Science and Technology(KAIST), Daejeon 34141 Republic of Korea (email: yongseop@kaist.ac.kr).

I. Kweon is with the Department of Electrical Engineering, Korea Advanced Institute of Science and Technology(KAIST), Daejeon 34141 Republic of Korea (e-mail: iskweon77@kaist.ac.kr).

S. B. Choi is with the Department of Mechanical Engineering, Korea Advanced Institute of Science and Technology(KAIST), Daejeon 34141 Republic of Korea (e-mail: sbchoi@kaist.ac.kr). speed in the perspective of Earth fixed frame with the accuracy of few centimeters using RTK [2], [3]. However, GNSS receiver is only able to measure the physical quantities of the mounting point of the antenna, which are not sufficient to reconstruct the dynamics of the vehicle including the sideslip angle.

Several approaches have been proposed to overcome the limitation of GNSS as a vehicle dynamics sensor. Inertial navigation system(INS) expands GNSS measurement into a threedimensional motion estimation including vehicle position, velocity, and attitude, by integrating accelerations and angular velocities measured from inertial measurement unit(IMU) [4]. Furthermore, combining odometer-derived velocity, the performance and reliability of INS are significantly improved even with GNSS signal outages [5], [6].

On the other hand, the motion estimation algorithms using ADAS sensors such as camera, LiDAR, or RADAR, have been developed widely. Visual odometry(VO) is one of the remarkable achievements that estimates the motion of camera only from successive image frames [7], [8]. Moreover, the estimation performance for motion tracking and scale ambiguity is drastically improved by bridging the inter-frame gap with IMU measurement [9], [10]. In a similar way, replacing feature points on images with point cloud data, a LiDAR version of motion estimation algorithm also has been introduced [11]. In the case of RADAR, despite its degraded resolution compared to LiDAR, there are several studies proposed to explore its usage as a motion sensor, with the advantage of the simultaneous measurement of range and velocity, and the robustness against operating condition [12], [13].

Meanwhile, the methods mentioned above estimate the motion, only relying on their own measurement, the actual 
motion of land vehicles follows the vehicle dynamics model constrained to the ground. Consequently, by combining the vehicle dynamics model with the motion sensor, the comprehensive motion estimation gives benefits for the improved performance and robustness [14]-[17].

The main drawback of the model-based estimator is that the model parameters used in vehicle dynamics are hard to be determined. Even though there are alternative methods replacing whole vehicle dynamics with neural network model [18], [19], there are remaining needs of exact identification of the model parameters in the view of vehicle control applications. In order to resolve this limitation, and identify adequate model parameters, various approaches have been proposed.

The longitudinal tire stiffness can be calculated from the wheel dynamics [20], [21], and the lateral tire stiffness also can be estimated based on the relationship between the sideslip angle and tire force [22]-[25]. For the vehicle inertial parameters, such as mass, moment of inertia, and axle distance from $\mathrm{CoG}$, recursive system identification methods based on the single track model are introduced [26]-[28].

Most of the identification algorithms are constructed based on the physical quantities measured at the position of vehicle CoG. However, in practice, the motion sensor is usually not mounted on vehicle $\mathrm{CoG}$, due to technical reasons. The distance between the motion sensor and vehicle CoG, called a lever arm, induces an addictive motion on the measurement and needs to be compensated when merging the sensor measurement with the vehicle model.

The non-holonomic constraint(NHC) assumption is a conventional way used to eliminate the effect of the lever arm. It assumes that there is no slip occurs on the rear axle, thus the lateral and vertical velocities at the rear axle are zero [29], [30]. However, adopting NHC into the vehicle model parameter identification can lead to erroneous results, since the vehicle dynamics model is derived from the tire force generated by slip, which directly conflicts with NHC assumption.

Alternatively, a dual Kalman filter approach using a virtual sensor measurement was introduced [31], [32]. This method establishes a Kalman filter to accomplish an indirect measurement of motion at vehicle $\mathrm{CoG}$, from remotely mounted IMU, while another Kalman filter estimates the vehicle model parameter using the virtual measurement, simultaneously. However, this method also has the prerequisite that the location of the sensor should be specified with respect to the vehicle body frame when implementing the virtual measurement.

In this paper, we introduce a vehicle model parameter identification method using remote mounted motion sensor for the following fundamental vehicle model parameters: the longitudinal position of $\mathrm{CoG}$, the sensor lever arm from CoG, the longitudinal and lateral tire stiffness, and the radius of gyration of vehicle. A single track model modified with a linearized tire model for the longitudinal and lateral dynamics is constructed, and the corresponding measurement model for remote mounted motion sensor is also presented. The proposed dynamics model and measurement model are integrated via unscented Kalman filter(UKF). The proposed identification algorithm is evaluated with numerical simulations and vehicle experiments in mild driving conditions.

\section{Vehicle And Sensor Model}

In this section, we introduce the equations of motion for the simplified vehicle planar dynamics model, and the corresponding measurement influence due to the mounting position of motion sensors with respect to vehicle CoG.

Assuming the vehicle chassis as a rigid body, the NewtonEuler equations of motion for the vehicle on a plane become

$$
\begin{gathered}
F_{x}=m \dot{v}_{x}-m r v_{y} \\
F_{y}=m \dot{v}_{y}+m r v_{x} \\
M_{z}=\dot{r} I_{z}
\end{gathered}
$$

When the lateral movement of the vehicle, $v_{y}$ is relatively small compared to the forward velocity $v_{x}$, we can assume that

$$
\begin{gathered}
v_{x}=v \cos \beta \approx v \\
v_{y}=v \sin \beta \approx v \beta
\end{gathered}
$$

where $\beta$ is the sideslip angle, defined by $\beta \approx \frac{v_{y}}{v_{x}}$, and $v$ is the planar speed of the vehicle. Then, the longitudinal and lateral forces acting on the vehicle can be represented in terms of $\beta$ as follows.

$$
\begin{gathered}
F_{x} \approx m \dot{v}-m r v \beta \\
F_{y} \approx m \dot{v} \beta+m v(r+\dot{\beta})
\end{gathered}
$$

When the aerodynamic effect is negligible, the only force acting on a vehicle is the friction between the ground and tires. Due to the highly nonlinear characteristics of tires, there are numerous parameters that affect the tire force. However, the tire force can be simplified with a linear coefficient when the contact surface to the ground is nearly in stick condition. In the following, we will describe the details of the vehicle dynamics model using the linear tire model, and how it manifests itself in the sensor measurement.

\section{A. Longitudinal Dynamics}

When a vehicle accelerates or decelerates, there are speed differences between tires and the ground speed developed. The longitudinal slip ratio of a tire is defined as

$$
\kappa=\frac{R \omega_{w h e e l}-v_{x}}{v_{x}}
$$

where $R$ is the nominal radius of the tire, and $\omega_{\text {wheel }}$ is the angular velocity measured from vehicle's wheel speed sensor.

The longitudinal force between a tire and the ground depends on the vertical load $F_{z}$ and the slip ratio $\kappa$. As shown in Fig. 1, the normalized force is proportional to the slip ratio in a small slip region and saturated when the slip ratio exceeds a certain slip ratio. Hence, in a mild driving maneuver, the longitudinal force (1) can be written as

$$
\begin{gathered}
F_{x i}=C_{\kappa i}\left(\kappa_{i}, F_{z i}\right) \cdot \kappa_{i} \\
C_{\kappa i} \approx C_{\kappa} F_{z i}
\end{gathered}
$$

where $C_{\kappa}$ is the normalized longitudinal stiffness of tires. 


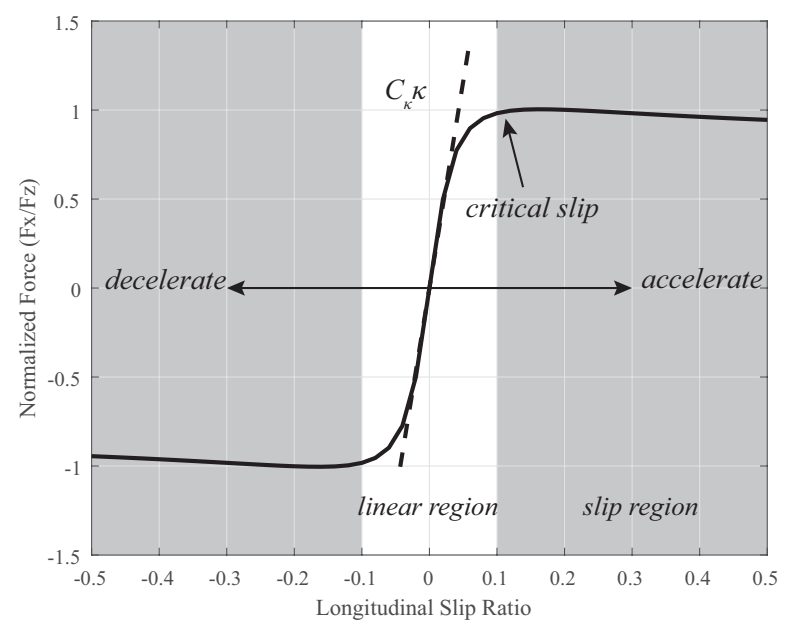

Fig. 1. Normalized longitudinal tire force with respect to tire slip ratio

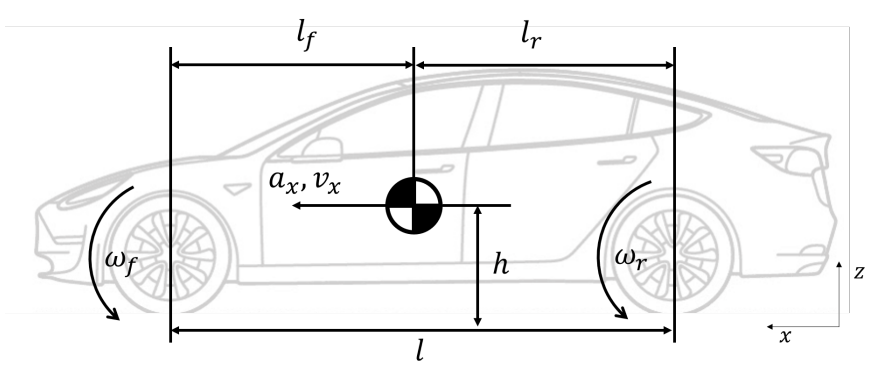

Fig. 2. Longitudinal vehicle model

Considering that a vehicle commonly uses the same tires on all wheels, and moves on a uniform surface, the longitudinal forces acting on the front and rear axle can be derived with the vertical load and the slip ratio. The vertical load of each axle is determined by the position of $\mathrm{CoG}$, and the dynamic load transfer due to the longitudinal acceleration,

$$
\begin{aligned}
& F_{z f}=m\left(g \frac{l_{r}}{l}-a_{x} \frac{h}{l}\right) \\
& F_{z r}=m\left(g \frac{l_{f}}{l}+a_{x} \frac{h}{l}\right)
\end{aligned}
$$

where $l$ refers the wheel base length, $l_{r}$ and $l_{f}$ refer the distances from $\mathrm{CoG}$ to front and rear axles, and $\mathrm{h}$ refers the height of CoG.

Combining (9)-(12), the longitudinal force generated by the front and rear tires becomes the function of the longitudinal slip ratio $\kappa$ with the nominal longitudinal tire stiffness $C_{\kappa}$.

$$
\begin{aligned}
& F_{x f}=m C_{\kappa} \frac{\left(g l_{r}-a_{x} h\right)}{l} \kappa_{f} \\
& F_{x r}=m C_{\kappa} \frac{\left(g l_{f}+a_{x} h\right)}{l} \kappa_{r}
\end{aligned}
$$

Note that the longitudinal forces, (13) and (14), refer the forces acting on each axle, not per tire, hence we use the average of $\kappa$ of two tires on the same axle.

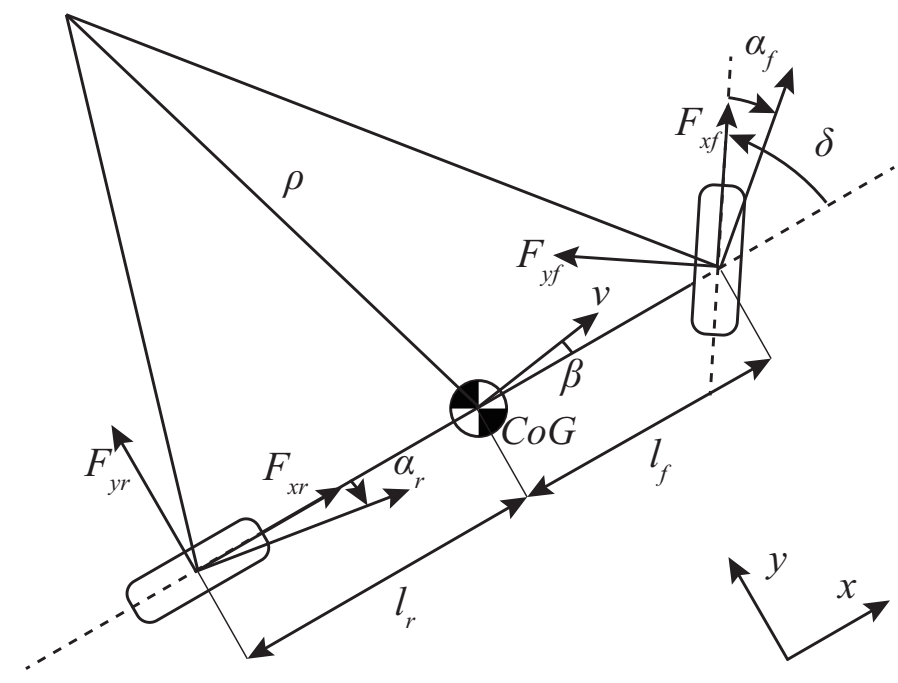

Fig. 3. Single Track Model Geometry

\section{B. Lateral Dynamics}

The single track model(also known as "bicycle model") assumes that the lateral dynamics of a vehicle can be described by the equivalent single tire per axle, while the roll dynamics including the lateral load transfer is neglected. Fig. 3 shows the geometry of the single track model when the vehicle turns with a given radius $\rho$.

From the equations of motion of the vehicle, (1)-(3), the single track model approximation of vehicle dynamics can be derived. We can rewrite (2) and (3) in terms of the front and rear tire force $F_{y f}$ and $F_{y r}$ as follows,

$$
\begin{aligned}
F_{y} & =F_{y f}+F_{y r} \\
M_{z} & =l_{f} F_{y f}-l_{r} F_{y r}
\end{aligned}
$$

In the same manner as the previous section, it is known that the lateral force of tire is proportional to the sideslip angle of tire $\alpha$, with the lateral tire stiffness(also known as "cornering stiffness"), $C_{\alpha i}$,

$$
F_{y i}=-C_{\alpha i}\left(\alpha_{i}, F_{z i}\right) \cdot \alpha_{i}
$$

where the sideslip angle of each tire $\alpha_{i}$ can be calculated by

$$
\begin{aligned}
& \alpha_{f}=\beta+l_{f} \frac{r}{v}-\delta \\
& \alpha_{r}=\beta-l_{r} \frac{r}{v}
\end{aligned}
$$

with the front steering angle $\delta$.

Substituting (15)-(19) into (2)-(3), the single track model for the sideslip $\beta$ and yaw rate $r$ is derived as below.

$$
\left[\begin{array}{c}
\dot{\beta} \\
\dot{r}
\end{array}\right]=\left[\begin{array}{cc}
-\frac{C_{\alpha f}+C_{\alpha r}}{m v_{x}} & -\frac{l_{f} C_{\alpha f}-l_{r} C_{\alpha r}}{m v_{x}^{2}}-1 \\
-\frac{l_{f} C_{\alpha f}-l_{r} C_{\alpha r}}{I_{z}} & -\frac{l_{f}^{2} C_{\alpha f}+l_{r}^{2} C_{\alpha r}}{I_{z} v_{x}}
\end{array}\right]\left[\begin{array}{c}
\beta \\
r
\end{array}\right]+\left[\begin{array}{c}
\frac{C_{\alpha f}}{m v_{x}} \\
\frac{l_{f} C_{\alpha f}}{I_{z}}
\end{array}\right] \delta
$$

In general, the lateral tire stiffness can be approximated as a constant when the effect of lateral load transfer is negligible.

$$
\begin{aligned}
& C_{\alpha f}=C_{\alpha} F_{z f} \\
& C_{\alpha r}=C_{\alpha} F_{z r}
\end{aligned}
$$


where $C_{\alpha}$ is the normalized lateral tire stiffness, which remains constant locally on the homogeneous road surface. Then, the linear form of the lateral tire force can be established as follows,

$$
\begin{aligned}
& F_{y f}=-m C_{\alpha} \frac{\left(g l_{r}-a_{x} h\right)}{l}\left(\beta+r l_{f}-\delta\right) \\
& F_{y r}=-m C_{\alpha} \frac{\left(g l_{f}+a_{x} h\right)}{l}\left(\beta-r l_{r}\right)
\end{aligned}
$$

and the single track model in (20) is reduced into

$$
\begin{aligned}
& {\left[\begin{array}{c}
\dot{\beta} \\
\dot{r}
\end{array}\right]=\left[\begin{array}{cc}
-\frac{C_{\alpha}}{v} g & \frac{C_{\alpha}}{v^{2}} a_{x} h-1 \\
\frac{1}{k^{2}} C_{\alpha} a_{x} h & -\frac{1}{k^{2}} \frac{C_{\alpha}\left(g l_{f} l_{r}-\left(l_{f}-l_{r}\right) a_{x} h\right)}{v}
\end{array}\right]\left[\begin{array}{l}
\beta \\
r
\end{array}\right]} \\
& +\left[\begin{array}{c}
\frac{C_{\alpha}}{v^{l}}\left(g l_{r}-a_{x} h\right) \\
\frac{1}{k^{2}} \frac{C_{\alpha} l_{f}}{l}\left(g l_{r}-a_{x} h\right)
\end{array}\right] \delta
\end{aligned}
$$

where $\bar{k}$ is the radius of gyration, defined as follows.

$$
I_{z}=m \bar{k}^{2}
$$

\section{Remote Mounted Sensor Kinematics}

The acceleration of the vehicle $\mathrm{CoG}$ can be expressed by sum of tire forces.

$$
\begin{aligned}
& m a_{x}=F_{x f}+F_{x r}-F_{y f} \delta \\
& m a_{y}=F_{y f}+F_{y r}
\end{aligned}
$$

Note that the longitudinal acceleration contains $F_{y f}$, whereas the lateral acceleration does not. Since this research assumes the vehicle driven in a steady maneuver, harsh braking or acceleration on a curved road is not considered. However, even the vehicle moves with a stationary turn, the lateral force of the front tire can be applied along longitudinal direction under tight turns.

The measurement from remote mounted sensors from vehicle $\mathrm{CoG}$ is influenced by the lever arm effect, induced by the motion of the vehicle. With the longitudinal distance between the sensor and vehicle $\mathrm{CoG}, l_{s}$, the sensor measurement becomes

$$
\begin{aligned}
r_{s} & =r \\
a_{x, s} & =a_{x}-l_{s} r^{2}+g \theta \\
a_{y, s} & =a_{y}+l_{s} \dot{r}+g \phi
\end{aligned}
$$

where the subscript $s$ is for the quantity on sensor position, and $\theta$ and $\phi$ are vehicle pitch and roll angle, respectively. The corresponding measurement or estimation result of the sideslip angle $\beta_{s}$, based on the remote mounted sensor, also becomes as below.

$$
\beta_{s}=\beta+l_{s} \frac{r}{v}
$$

Combining the linear tire force models denoted in previous sections, (13)-(14) and (23)-(24), and the sensor measurement models, (27)-(32), the acceleration measurements from the

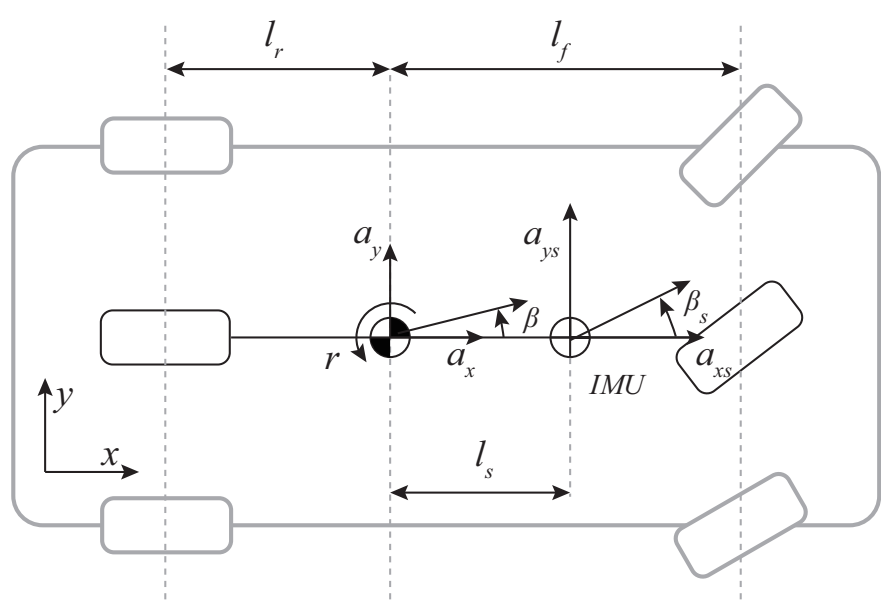

Fig. 4. Remote Mounted Sensor on a Vehicle

remote sensor are

$$
\begin{aligned}
a_{x, s}= & C_{\kappa}\left(\frac{g l_{r}-a_{x} h}{l} \kappa_{f}+\frac{g l_{f}+a_{x} h}{l} \kappa_{r}\right) \\
& -C_{\alpha} \frac{g l_{r}-a_{x} h}{l} \alpha_{f} \delta+r v\left(\beta_{s}-l_{s} \frac{r}{v}\right)+g \theta \\
a_{y, s}= & C_{\alpha}\left(-g \beta_{s}+\left(g l_{s}+a_{x} h\right) \frac{r}{v}+\left(g l_{r}-a_{x} h\right) \frac{\delta}{l}\right)+g \phi
\end{aligned}
$$

and the single track model, in terms of the sensor measurements, is represented with the following form.

$$
\begin{aligned}
{\left[\begin{array}{c}
\dot{\beta}_{s} \\
\dot{r}_{s}
\end{array}\right]=} & {\left[\begin{array}{cc}
-\frac{C_{\alpha}}{v} g & \frac{C_{\alpha}}{v} a_{x} h-1 \\
\frac{1}{k^{2}} C_{\alpha} a_{x} h & -\frac{1}{k^{2}} \frac{C_{\alpha}\left(g l_{f} l_{r}-\left(l_{f}-l_{r}\right) a_{x} h\right)}{v}
\end{array}\right]\left[\begin{array}{c}
\beta_{s}-l_{s} \frac{r}{v} \\
r
\end{array}\right] } \\
& +\left[\begin{array}{c}
\frac{C_{\alpha}}{v l}\left(g l_{r}-a_{x} h\right) \\
\frac{1}{k^{2}} \frac{C_{\alpha} l_{f}}{l}\left(g l_{r}-a_{x} h\right)
\end{array}\right] \delta+\left[\begin{array}{c}
\dot{r} \frac{l_{s}}{v} \\
0
\end{array}\right]
\end{aligned}
$$

The derivative of sideslip angle, $\dot{\beta}_{s}$, can be formulated based on the kinematic relationship:

$$
\dot{\beta}_{s, k i n}=\frac{a_{y, s}-g \phi}{v}-r
$$

Although the sensor has the capability to measure the sideslip angle directly, the system becomes highly sensitive when it depends on velocity measurements which can suffer external disturbances such as GNSS signal outage. Hence, alternatively, the kinematic model, (36), is adopted to obtain the time derivative of sideslip angle.

\section{Parameter Identification Filter}

The vehicle model provided in the previous section contains vehicle-specific parameters such as the tire stiffness, position of CoG, and sensor lever arm, which are normally hard to determine. In order to achieve reconstructing the vehicle dynamics model, and validate the potential capability for complementary performance enhancement through sensor fusion from additionally mounted sensors, an online parameter identification filter is suggested.

The identification process of vehicle model parameters is based on the Kalman filter, which is widely used to obtain 
the optimal solution for rank deficient systems. Originally, the Kalman filter is constructed for linear-time-invariant systems, thus the prediction and correction model need to be in the form of linear combinations of states.

For non-linear systems, an extended version of the Kalman filter is developed, which performs a linearization of nonlinear models around recently estimated states for each step. The EKF approximates the system dynamics as the first-order Taylor approximation, and the probability density as a Gaussian distribution, which requires the numerical calculation of Jacobian matrices at every update.

As the degraded performance of EKF of a system with the probability distribution of a random vector, the UKF was proposed [33], [34]. The UKF is based on the unscented transform, which gives the accuracy up to a second-order Taylor approximation, whereas the computational effort has the same order $O\left(n^{3}\right)$ as that of the EKF. Additionally, the estimation architecture of the UKF does not require the derivatives, which brings convenience to the implementation of practical systems.

In the rest of this section, the identifiability analysis and the UKF formulation for the vehicle model parameter estimation will be demonstrated.

\section{A. System Identifiability}

The unknown vehicle model parameters, $l_{r}, l_{s}, C_{\alpha}, C_{\kappa}$, and $\bar{k}$, need to be determined to comprise the vehicle dynamics model given in the previous section. The state vector and available measurements used to establish identification filter are as follows, respectively,

$$
\begin{aligned}
\hat{\mathbf{x}} & =\left[\begin{array}{lllll}
\hat{l}_{r} & \hat{C}_{\kappa} & \hat{C}_{\alpha} & \hat{l}_{s} & \hat{\bar{k}}
\end{array}\right]^{\top} \\
\mathbf{z} & =\left[\begin{array}{llll}
\dot{\beta}_{s, k i n} & \dot{r} & a_{x, s} & a_{y, s}
\end{array}\right]^{\top}
\end{aligned}
$$

and the corresponding measurement model derived with the vehicle dynamics model, (33)-(35), can be represented in terms of the filter state,

$$
\begin{aligned}
\hat{\mathbf{z}} & =h(\hat{\mathbf{x}}, \mathbf{u}) \\
& =\left[\begin{array}{c}
-\frac{g}{v} \hat{C}_{\alpha}\left(\beta_{s}-\hat{l}_{s} \frac{r}{v}\right)+\frac{a_{r} h}{v^{2}} \hat{C}_{\alpha} r-r+\frac{g \hat{l}_{r}-a_{x} h}{v l} \hat{C}_{\alpha} \delta+\dot{r} \frac{\hat{l}_{s}}{v} \\
\frac{1}{k^{2}}\left(a_{x} h\left(\beta_{s}-\hat{l}_{s} \frac{r}{v}\right)-\left(\hat{l}_{f} \hat{l}_{r} g-\left(\hat{l}_{f}-\hat{l}_{r}\right) a_{a} h\right) \frac{r}{v}+\hat{l}_{f}\left(g \hat{l}_{r}-a_{x} h\right) \frac{\delta}{\tau}\right) \\
\hat{C}_{\kappa}\left(\frac{g \hat{l}_{r}-a_{x} h}{l} \kappa_{f}+\frac{g \hat{g}_{f}+a_{x} h}{l} \kappa_{r}\right)-\hat{C}_{\alpha} \frac{g \hat{l}_{r}-a_{x} h}{l} \alpha_{f} \delta+r v\left(\beta_{s}-\hat{l}_{s} \frac{r}{v}\right)+g \theta \\
\hat{C}_{\alpha}\left(-g \beta_{s}+\left(g \hat{l}_{s}+a_{x} h\right) \frac{r}{v}+\left(g \hat{l}_{r}-a_{x} h\right) \frac{\delta}{2}\right)+g \phi+\dot{r} \hat{l}_{s}
\end{array}\right]
\end{aligned}
$$

where the hat operator denotes a estimated value.

The structural identifiability for the given system is evaluated by investigating the identifiability matrix, $\mathcal{O}_{I}(\mathbf{x}, \mathbf{u})$ defined by

$$
\mathcal{O}_{I}=\left(\begin{array}{c}
\frac{\partial}{\partial \mathbf{x}} h(\mathbf{x}, \mathbf{u}) \\
\frac{\partial}{\partial \mathbf{x}} \mathcal{L} h(\mathbf{x}, \mathbf{u}) \\
\vdots \\
\frac{\partial}{\partial \mathbf{x}} \mathcal{L}^{n-1} h(\mathbf{x}, \mathbf{u})
\end{array}\right)
$$

where the operator, $\mathcal{L}^{i}$, denotes the $i$ th order extended Lie derivative defined by

$$
\mathcal{L}^{i} h(\mathbf{x}, \mathbf{u})=\sum_{j=0}^{\infty} \frac{\partial \mathcal{L}^{i-1} h(\mathbf{x}, \mathbf{u})}{\partial \mathbf{u}^{(j)}} \mathbf{u}^{(j+1)}
$$

where $\mathbf{u}^{(j)}$ is the $j$ th time derivative of $u$ [35]. If the system with $n_{\mathbf{x}}$ independent states satisfies $\operatorname{rank}\left(\mathcal{O}_{I}(\mathbf{x}, \mathbf{u})\right)=n_{\mathbf{x}}$, then the model is locally structurally identifiable [36], [37].

Although the first order Jacobian matrix for the given system, $\frac{\partial}{\partial \mathbf{x}} h(\mathbf{x}, \mathbf{u})$, has the rank deficiency, the extended Lie derivative of $h(\mathbf{x}, \mathbf{u})$ implies full column rank of $\mathcal{O}_{I}$, with the consideration of the system input $\mathbf{u}=\left[\begin{array}{lll}\beta_{s} & r & \delta\end{array}\right]$. It should be noted that the system only has full rank with non-zero excitation of the lateral movement, hence the update process should be limited to the longitudinal parameters when the vehicle moves in a straight trajectory.

\section{B. UKF Formulation}

The parameter estimation proposed in this research is performed by the UKF. The key difference between the UKF and the EKF is the approximation method for nonlinear models. The UKF linearizes the system by the statistical linearization method, based on the unscented transform. This method approximates the system with a linear regression over $2 n+1$ point, chosen by the prior distribution of the states. These points are called the sigma points, given by the following transformation

$$
\chi_{i}= \begin{cases}\hat{\mathbf{x}}_{k \mid k-1} & \text { if } i=0 \\ \hat{\mathbf{x}}_{k \mid k-1}+\sqrt{(n+\lambda) \Sigma_{k \mid k-1}} i & \text { if } 0<i \leq n \\ \hat{\mathbf{x}}_{k \mid k-1}-\sqrt{(n+\lambda) \Sigma_{k \mid k-1}} i & \text { if } n<i \leq 2 n\end{cases}
$$

where $\chi, \Sigma$, and $\lambda$ denote the sigma points, prior states covariance, and a scaling parameter, given by $\lambda=a^{2}(n+k)-n$ with tuning parameters $a$ and $k$, which determine the spread of the sigma points. The subscript $i \mid j$ denotes that the estimated parameter in $i$ th step using the information of $j$ th step.

The corresponding weights for each point are

$$
\begin{aligned}
\mathcal{W}_{i}^{c} & = \begin{cases}\frac{\lambda}{n+\lambda}+\left(1-a^{2}+b\right) & \text { if } i=0 \\
\frac{1}{2(n+\lambda)} & \text { if } i \neq 0\end{cases} \\
\mathcal{W}_{i}^{m} & = \begin{cases}\frac{\lambda}{n+\lambda} & \text { if } i=0 \\
\frac{1}{2(n+\lambda)} & \text { if } i \neq 0\end{cases}
\end{aligned}
$$

where $\mathcal{W}_{i}^{c}$ and $\mathcal{W}_{i}^{m}$ are weights for the covariance and mean calculation, respectively.

The average measurement, $\hat{z}$ is obtained according to the measurement model, (39), over the sigma points:

$$
\begin{gathered}
\mathcal{Z}_{i}=h\left(\chi_{i}\right) \\
\hat{z}=\sum_{i=0}^{2 n} \mathcal{W}_{i}^{m} \mathcal{Z}_{i}
\end{gathered}
$$

Similarly, the error and state covariance are propagated as below

$$
\begin{aligned}
& S=\sum_{i=0}^{2 n} \mathcal{W}_{i}^{c}\left(\mathcal{Z}_{i}-\hat{z}\right)\left(\mathcal{Z}_{i}-\hat{z}\right)^{\top}+R \\
& \bar{\Sigma}=\sum_{i=0}^{2 n} \mathcal{W}_{i}^{c}\left(\chi_{i}-\chi_{0}\right)\left(\mathcal{Z}_{i}-\hat{z}\right)+Q
\end{aligned}
$$

where $R$ and $Q$ are the observation and process noise respectively. 
TABLE I

Vehicle Parameters used in Simulation

\begin{tabular}{llll}
\hline \hline B-Class Sports Car & Parameter & Value & Unit \\
\hline Vehicle mass & $m$ & 1140 & $\mathrm{~kg}$ \\
Moment of inertia & $I_{z}$ & 1320 & $\mathrm{~kg} \mathrm{~m}^{2}$ \\
Radius of gyration & $\bar{k}$ & 1.1 & $\mathrm{~m}$ \\
Wheelbase & $l$ & 2.33 & $\mathrm{~m}$ \\
Front axle distance & $l_{f}$ & 1.165 & $\mathrm{~m}$ \\
Rear axle distance & $l_{f}$ & 1.165 & $\mathrm{~m}$ \\
$\quad$ CoG Height & $h$ & 0.5 & $\mathrm{~m}$ \\
$\quad$ Nominal lateral tire stiffness & & & \\
$\quad \sim|\alpha|<0.1$ & $C_{\alpha}$ & 15 & $1 / \mathrm{rad}$ \\
$\quad \begin{array}{l}\text { Nominal longitudinal tire stiffness } \\
\quad|\kappa|<0.1\end{array}$ & & & \\
$\quad C_{\kappa}$ & 19 & $1 /$ (slip ratio) \\
Sensor lever arm & $l_{s}$ & 0.5 & $\mathrm{~m}$ \\
\hline
\end{tabular}

The state correction is performed when a new set of measurement is available:

$$
\begin{aligned}
K & =\bar{\Sigma} S^{-1} \\
\hat{\mathbf{x}}_{k \mid k} & =\chi_{0}+K(z-\hat{z}) \\
\Sigma_{k \mid k} & =\bar{\Sigma}-K S K^{\top}
\end{aligned}
$$

Since the vehicle parameters are time-invariant states, the prior state is not updated without the measurement correction, $\hat{\mathbf{x}}_{k \mid k-1}=\hat{\mathbf{x}}_{k-1 \mid k-1}$, whereas the process noise, $Q$, is added into the state covariance, $\Sigma$, in the purpose of maintaining the estimation performance against potential violations of model assumptions or environmental changes.

\section{Simulation AND EXPERIMENTAL REsults}

The proposed identification of vehicle model parameters was evaluated by a numerical simulation. The aim of the simulation is investigating the performance of the identification algorithm, and exploring the effect of the initial state and tuning parameters. The simulation was performed on the CarSim, which is a industrial software for simulating and analyzing of the vehicle dynamics.

\section{A. Simulation Results}

All of the vehicle model parameters used for the simulation are summarized in Table I. The inertial and geometrical parameters are extracted from the vehicle model data, whereas the longitudinal and lateral tire stiffness are obtained using linear regression as shown in Fig. 5, since originally the stiffness is modeled as a function of the slip, vertical load, road surface friction, and elastic characteristics.

The simulations were made for various road shapes, but here the results of the representative scenario which contains several curves and straights are presented. The driving scenario is described in Fig. 6, considering the characteristics of realworld drivers in normal driving circumstances. According to the previous research for the driving pattern analysis [38], it had been shown that the majority of peak acceleration in a moderate driving scenario is $3-4 \mathrm{~m} / \mathrm{s}^{2}$. Therefore, the simulation driving maneuver is set to be limited to $3.5 \mathrm{~m} / \mathrm{s}^{2}$ for the longitudinal and lateral acceleration with the homogeneous road friction coefficient.

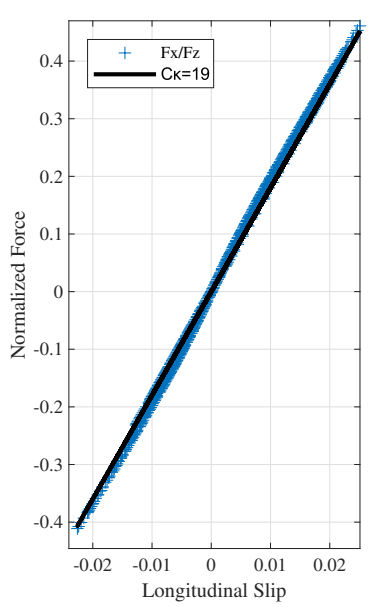

(a)

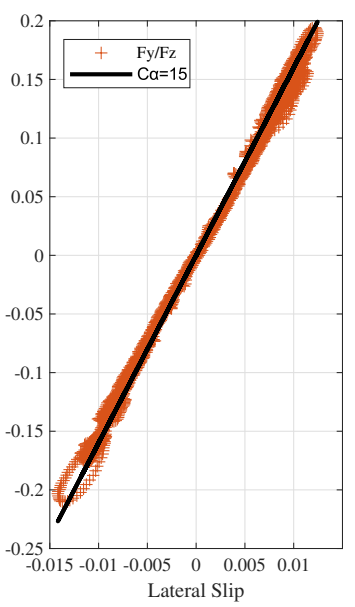

Fig. 5. Normalized tire force compared to linear model in the low-slip region: (a) Longitudinal normalized force versus $\kappa$ (b) Lateral normalized force versus $\alpha$
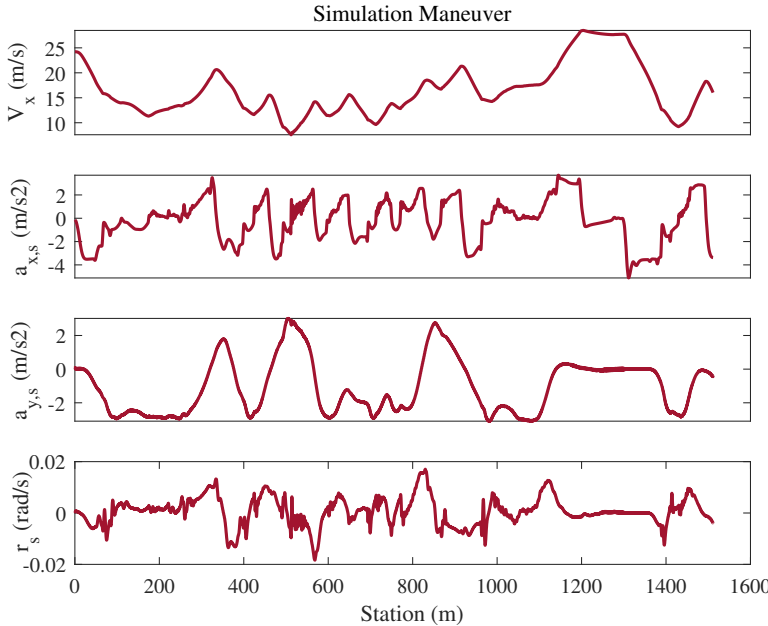

Fig. 6. Velocity and inertial measurements of the simulation scenario
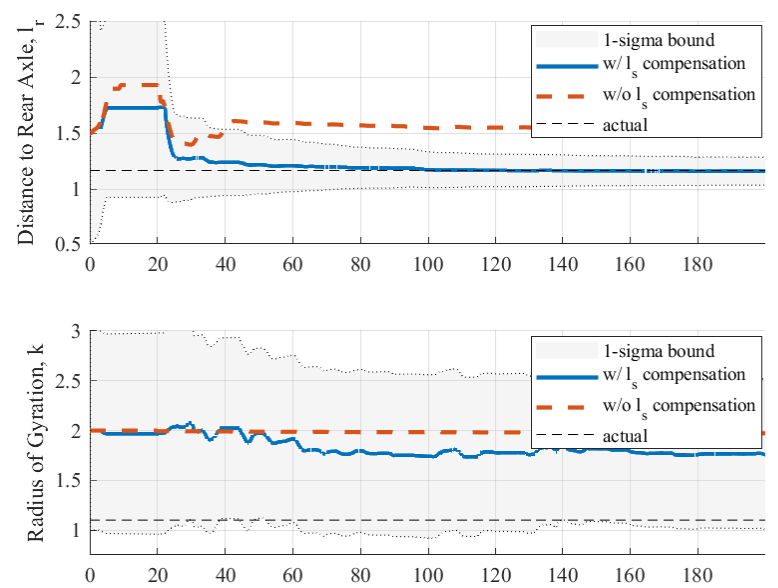

Fig. 7. Vehicle geometrical parameter identification result 

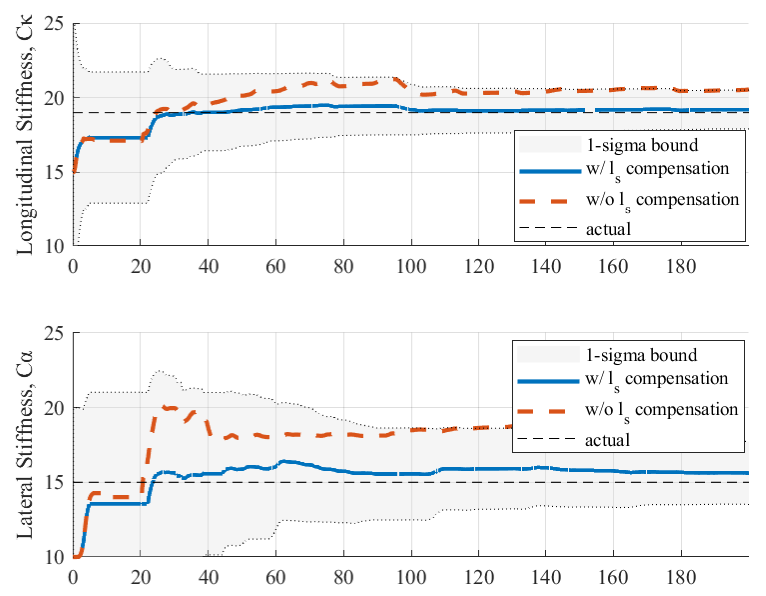

Fig. 8. Tire stiffness identification result
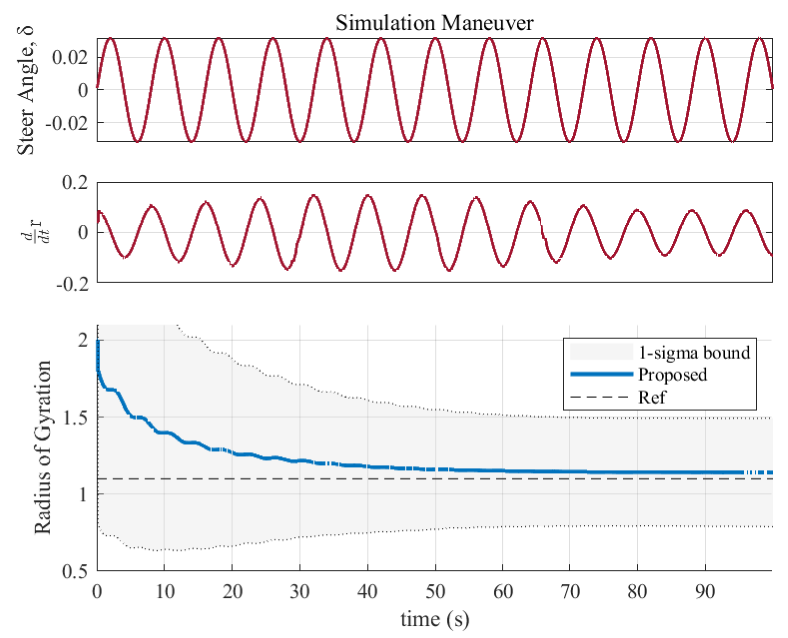

Fig. 9. Subsequent sine-wave steer scenario for the excitation signal of identifying radius of gyration.

Fig. 7-8 shows the identified vehicle parameters with and without the correction of the remote sensor lever arm effect. Without the simultaneous identification of the lever arm effect, the identified vehicle model converges to an incorrect direction to resolve the error from the lever arm, $l_{s}$, with the own dynamics of the vehicle model itself, whereas the estimation results with the consideration of the lever arm enhance the estimation performance of the vehicle parameters. However, it is worth noting that the radius of gyration is not well converging to the actual value, while other estimations tend to converge to the actual model. Since the given driving maneuver in the simulation is hardly restricted, the excitation condition for the identification of $\bar{k}$ is rarely occurred. Thus, we construct a subsequent scenario, which contains rotational excitation with sine-wave steer input. Fig. 9 shows the steer input and estimated $\bar{k}$ in additional maneuver.

The comparative result of the proposed method and NHC method for the sensor lever arm estimation is presented in Fig. 10. It can be found that even the vehicle moves with low-level acceleration, the sideslip angle of the rear axle, $\alpha_{r}$, violates

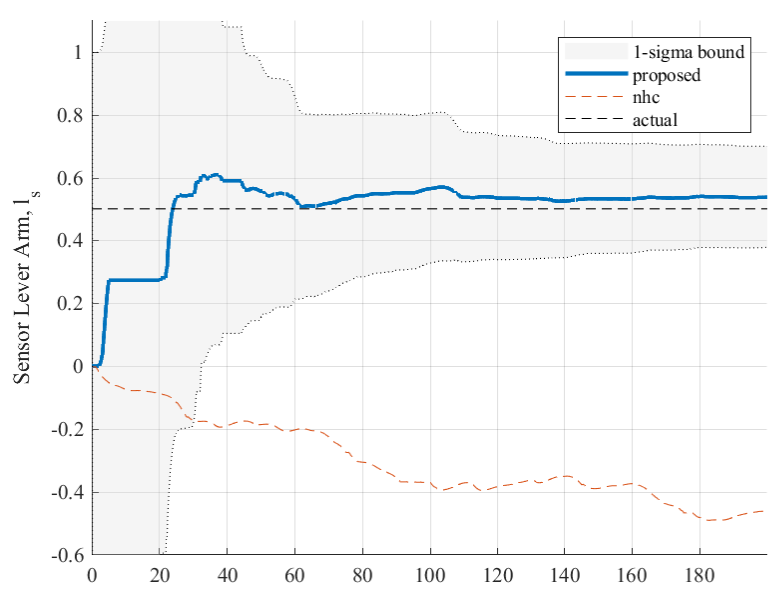

Fig. 10. Lever arm estimation results for remote mounted sensor
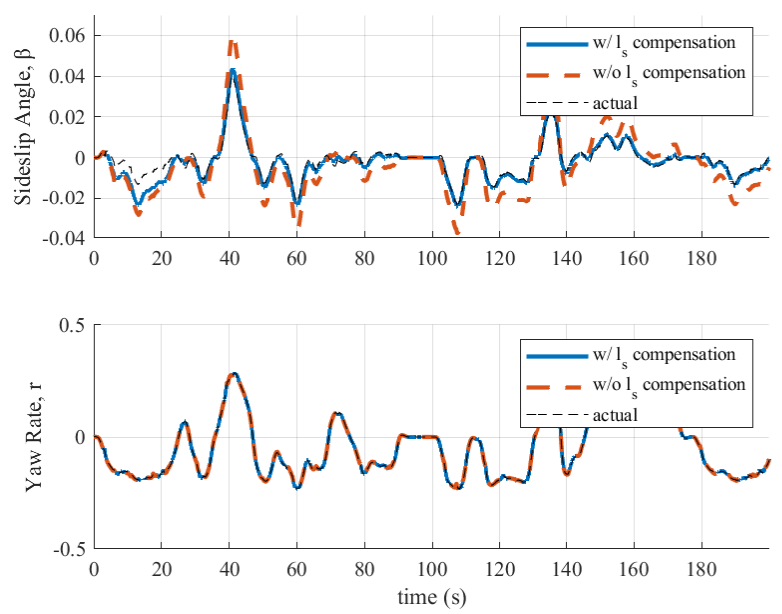

Fig. 11. Open-loop estimation of sideslip angle and yaw rate with simultaneous identification of model parameters

the non-slip assumption, thus the estimation result of the NHC method obviously leads to a wrong value. Meanwhile, the proposed method provides an acceptable estimation result based on the single track model.

Since the main purpose of the parameter identification is for the accurate control and estimation of the vehicle dynamics, the state estimation for the sideslip angle and yaw rate is conducted. The estimation follows a simple open-loop integration of the single track model, described in the previous section, with the estimated model parameters. As seen in Fig. 11 , the identified model well represents the actual dynamics of the given condition. The sideslip angle reconstructed from the model achieves a reliable tracking performance of the actual $\beta$, even during the combined slip region. However, the yaw rate is not much varying from the actual value. The measurement model used in this research directly includes the yaw rate measurement, and the lever arm effect does not implicate an interference on angular velocity measurements. As a result, the yaw rate estimation shows less model sensitivity regardless of the accuracy of model parameters. 

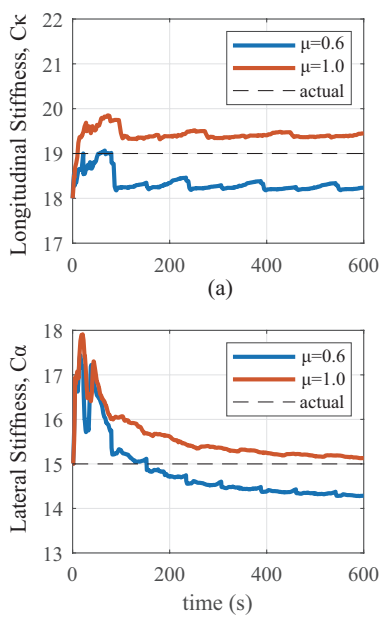

(b)

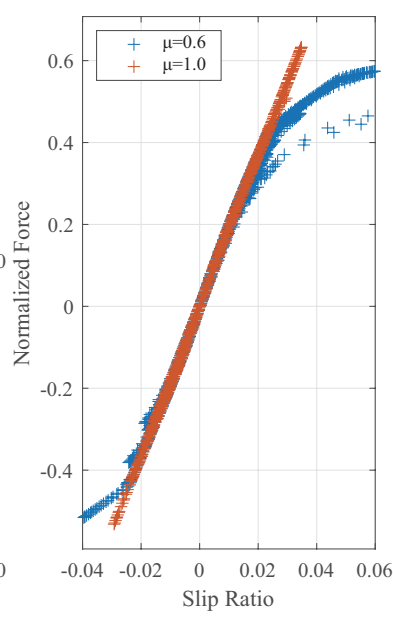

(c)

Fig. 12. Tire stiffness estimation on different types of road surface with same driving maneuver. (a)Longitudinal tire stiffness estimation. (b)Lateral tire stiffness estimation. (c)Force-slip curves for longitudinal normalized force.

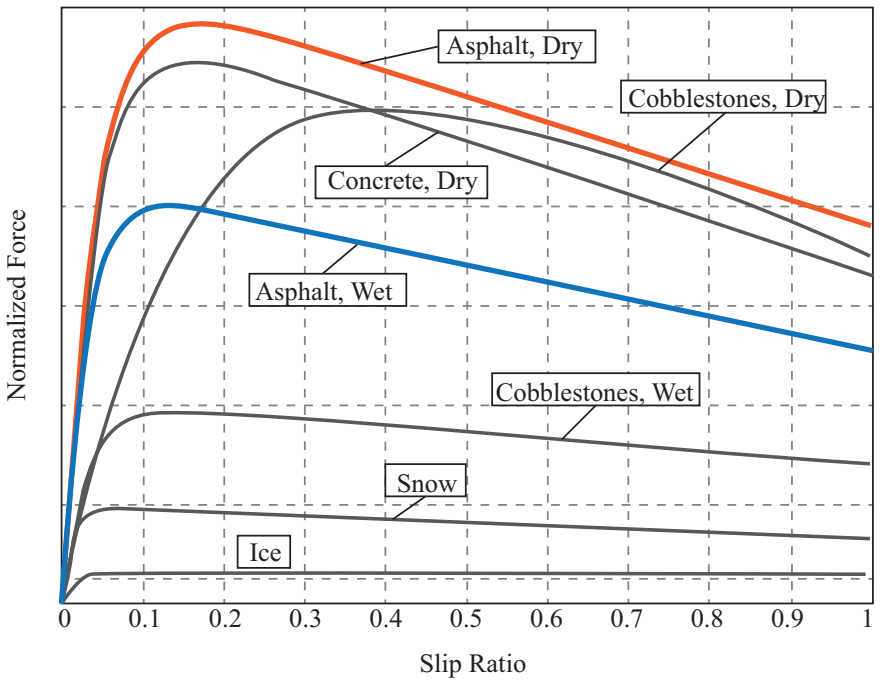

Fig. 13. Nominal curve for tire slip ratio and normalized force [39]; initial slopes for dry and wet pave road are almost same on linear region, whereas normalized force on wet surface(blue line) is saturated with smaller amount of slip than dry surface(orange line).

Additionally, the simulation was conducted both on the high and low surface friction condition, with the purpose of feasibility check for potential usage of proposed methods in the vehicle dynamics and control applications. The test consisted of two different surface conditions, $\mu=1.0$ and $\mu=0.6$, which are typical road friction coefficients for the dry pavement and wet pavement, respectively.

A comparison between the estimation results for different road friction is presented in Fig. 12(a) and Fig. 12(b). It can be seen the tire stiffness on the low friction surface has a lower value compared to that on the high friction surface. The tire behavior on different surfaces can be modeled by a method called friction similarity, which predicts the effect of the degraded friction coefficient while maintaining the initial response in the small slip region [40]. This phenomenon is

TABLE II

Vehicle Parameters used in Simulation

\begin{tabular}{llll}
\hline \hline 2016 Genesis G80 3.3 4WD & Parameter & Value & Unit \\
\hline Vehicle mass(Unloaded) & $m$ & 1998 & $\mathrm{~kg}$ \\
Moment of inertia(Unloaded) & $I_{z}$ & 4124 & $\mathrm{~kg} \mathrm{~m}$ \\
Radius of gyration(Unloaded) & $\bar{k}$ & 1.44 & $\mathrm{~m}$ \\
Wheelbase & $l$ & 3.01 & $\mathrm{~m}$ \\
Front axle distance(Unloaded) & $l_{f}$ & 1.57 & $\mathrm{~m}$ \\
Rear axle distance(Unloaded) & $l_{f}$ & 1.44 & $\mathrm{~m}$ \\
CoG Height(Unloaded) & $h$ & 0.52 & $\mathrm{~m}$ \\
$\quad \begin{array}{l}\text { Nominal lateral tire stiffness } \\
\quad \text { Typical value }\end{array}$ & & & \\
Nominal longitudinal tire stiffness & $C_{\alpha}$ & 10 & $1 / \mathrm{rad}$ \\
$\quad$ Typical value & $C_{\kappa}$ & 20 & $1 /$ (slip ratio) \\
Sensor lever arm & $l_{s}$ & -0.4 & $\mathrm{~m}$ \\
\hline \hline
\end{tabular}
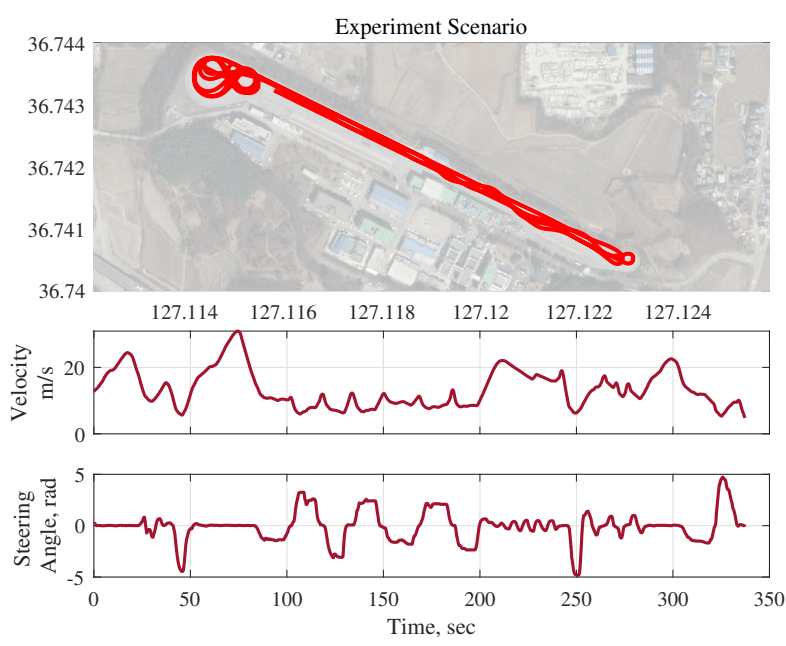

Fig. 14. Vehicle maneuver in experiment

demonstrated in Fig. 12(c). The normalized force on the low friction surface has a similar slope to that on the high friction surface when the slip ratio is nearly zero. However, on the low friction surface, an excessive slip is required to generate the same amount of force, which causes saturation on the tire force. This result implies a potential necessity of the estimation criteria for switching the estimation strategy according to dynamic states for the real environment implementation.

\section{B. Vehicle Experiment Results}

Although validation of the proposed algorithm was performed based on the numerical simulation, an experimental evaluation was conducted in order to secure the feasibility with the unpredictable disturbances which might occur in real driving situations. A passenger vehicle equipped with SINS was used in the experiments, and the available parameters for the experimental vehicle are as described in Table II.

The test scenario is as described in Fig. 14. The maneuver consists of several acceleration and braking in the straight line, and multiple loops of figure 8 shape turn which is intended to obtain the estimation result from a short period of driving fragments within a limited proving ground area.

However, unlike the simulation, the longitudinal slip ratio and the steering angle at the front wheels cannot be measured directly. The wheel speed measurements from in-vehicle 


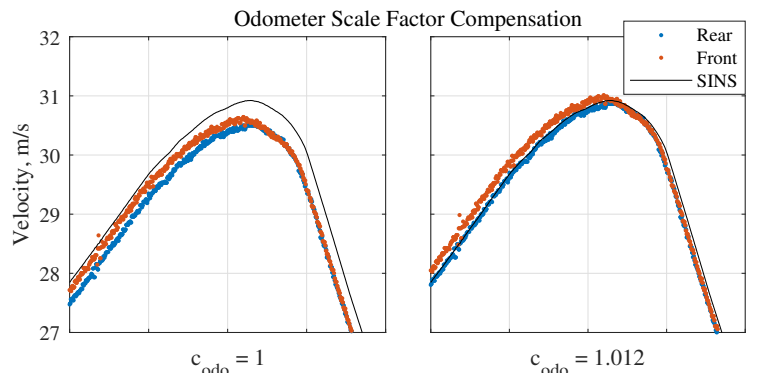

Fig. 15. Odometer scale compensation
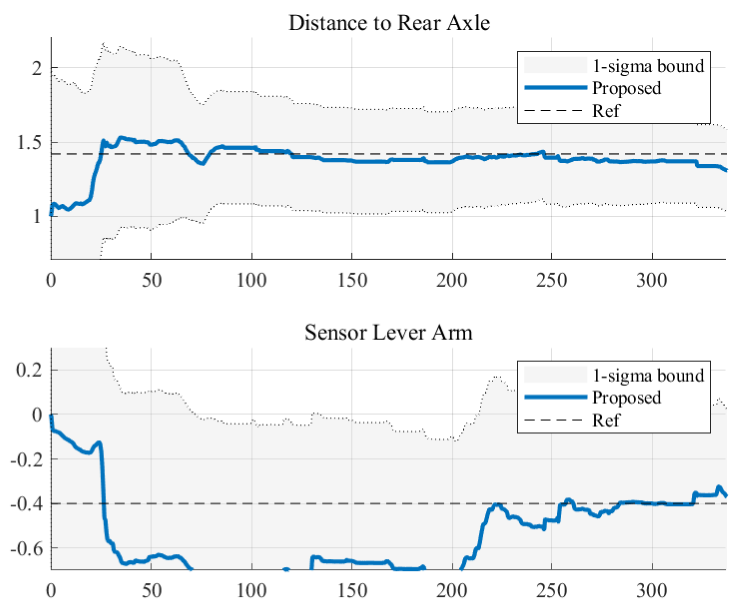

Fig. 16. Geometrical parameter identification results: (top) Vehicle CoG position identification. (bottom) Sensor lever arm identification

odometers have a scale error depending on the effective radius of tires, and the steering system also has its own kinematics which is manifested in the nonlinear response of the wheel angle with respect to the rotation of the steering wheel. Thus, a simple regression process was performed to specify the coefficients of the odometer scale and steering ratio.

A comparative result for the odometer scale factor compensation is given in Fig. 15. Without the compensation, the calculated longitudinal slip denotes continuous negative slip. Thus, in this experiment, the odometer measurement is compensated with a coefficient of 1.012, and similarly, the steering ratio is set to be 13.8 within the range $\left(-90^{\circ}, 90^{\circ}\right)$.

The identification results for the experimental vehicle is presented in Fig. 16-17. The overall estimation results seem to follow the ground truth, whereas there exist fluctuations in the first half of the experimental maneuver. The fluctuations can be interpreted as due to the excessive steering angle in the corresponding section. As it can be found in Fig. 14, the maximum steering angle in the first half of the experimental scenario exceeds $360^{\circ}$ which violates the assumption that the steering angle, $\delta$, is small enough to approximate.

However, for the tire stiffness, the ground truth of the longitudinal and lateral stiffness is still vague, while the identification results converge to certain values [41]. Thus, an indirect validation is established to ensure the performance of the proposed identification method.

Fig. 18 shows the open-loop estimation performance of the
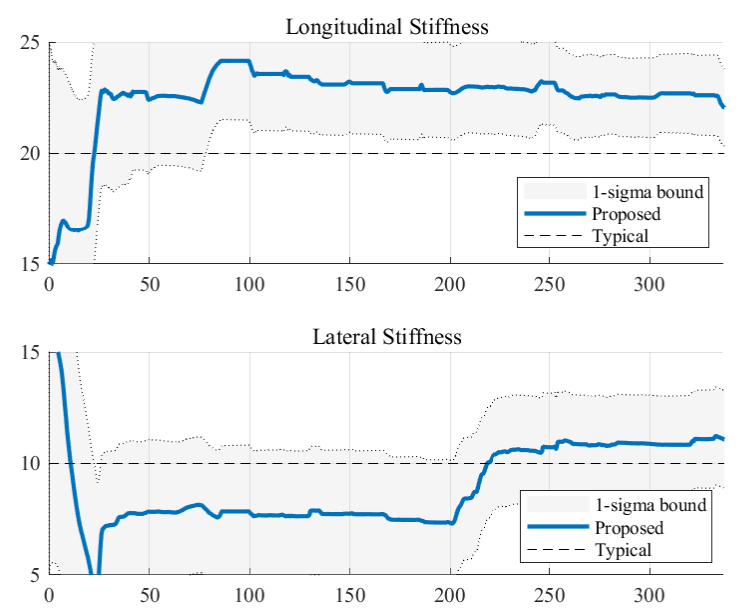

Fig. 17. Tire stiffness identification results: (top) Longitudinal tire stiffness. (bottom) Lateral tire stiffness
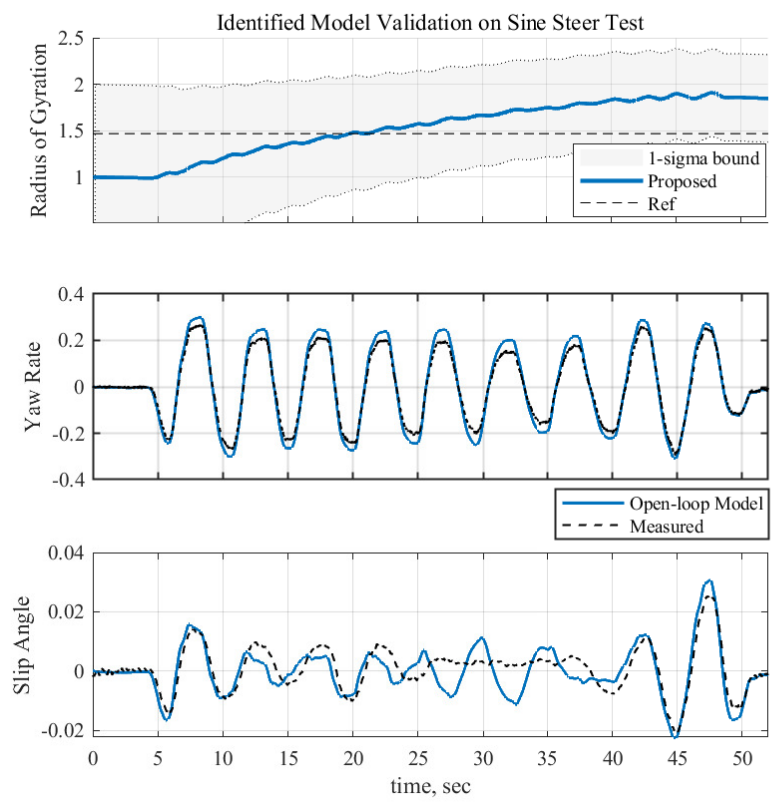

Fig. 18. Open-loop integration of identified model

identified model. The yaw rate and the sideslip angle seem to be following the measured value in the acceptable range without additional sensor feedback. The sideslip model has an erroneous region, from $30 s$ to $40 s$, where a braking is applied to the vehicle. Since the single track model assumes that the time derivative of longitudinal velocity is negligible, the model integration has its weakness with the speed change.

For the estimation result for the radius of gyration, $\bar{k}$ has been converged to a larger value than the unloaded moment of inertia. Although the moment of inertia of loaded vehicles cannot be measured, it is shown that the identified model well describes the motion of the vehicle, which is the primary purpose of the model identification. 


\section{CONCLUSION}

In this paper, a novel vehicle dynamics model identification method using a sensor installed at an arbitrary position. The proposed method gives the linearized tire stiffnesses, the position of $\mathrm{CoG}$, and the lever arm of measurements, which imply a capability to contribute to future researches, as a baseline for advanced state estimation of vehicle dynamics.

The proposed method is evaluated with both the simulation and the experiment, and the corresponding results show a stable and fast convergence with versatile conditions. Also, the only prior information about the target vehicle's parameter used in the identification process is a wheelbase, which can be easily obtained. This remarks the proposed method is widely applicable for various vehicle types.

\section{RefERENCES}

[1] V. K. Kukkala, J. Tunnell, S. Pasricha, and T. Bradley, "Advanced driver-assistance systems: A path toward autonomous vehicles," IEEE Consumer Electronics Magazine, vol. 7, no. 5, pp. 18-25, 2018.

[2] Q. C. Sun, J. C. Xia, J. Foster, T. Falkmer, and H. Lee, "Pursuing precise vehicle movement trajectory in urban residential area using multi-gnss rtk tracking," Transportation research procedia, vol. 25, pp. 2356-2372, 2017.

[3] M. Higuchi and N. Kubo, "Achievement of continuous decimeter-level accuracy using low-cost single-frequency receivers in urban environments," in Proceedings of the 29th International Technical Meeting of the Satellite Division of the Institute of Navigation (ION GNSS+ 2016), pp. 1891-1913, 2016.

[4] E. Abbott and D. Powell, "Land-vehicle navigation using gps," Proceedings of the IEEE, vol. 87, no. 1, pp. 145-162, 1999.

[5] Z. Li, J. Wang, B. Li, J. Gao, and X. Tan, "Gps/ins/odometer integrated system using fuzzy neural network for land vehicle navigation applications," The Journal of Navigation, vol. 67, no. 6, pp. 967-983, 2014.

[6] X. Niu, S. Nassar, and N. EL-SHEIMY, "An accurate land-vehicle mems imu/gps navigation system using 3d auxiliary velocity updates," Navigation, vol. 54, no. 3, pp. 177-188, 2007.

[7] D. Nistér, O. Naroditsky, and J. Bergen, "Visual odometry," in Proceedings of the 2004 IEEE Computer Society Conference on Computer Vision and Pattern Recognition, 2004. CVPR 2004., vol. 1, pp. I-I, Ieee, 2004.

[8] D. Nistér, O. Naroditsky, and J. Bergen, "Visual odometry for ground vehicle applications," Journal of Field Robotics, vol. 23, no. 1, pp. 3-20, 2006.

[9] L. Von Stumberg, V. Usenko, and D. Cremers, "Direct sparse visualinertial odometry using dynamic marginalization," in 2018 IEEE International Conference on Robotics and Automation (ICRA), pp. 25102517, IEEE, 2018.

[10] T. Qin, P. Li, and S. Shen, "Vins-mono: A robust and versatile monocular visual-inertial state estimator," IEEE Transactions on Robotics, vol. 34, no. 4, pp. 1004-1020, 2018.

[11] J. Zhang and S. Singh, "Loam: Lidar odometry and mapping in realtime.," in Robotics: Science and Systems, vol. 2, 2014.

[12] Y. Almalioglu, M. Turan, C. X. Lu, N. Trigoni, and A. Markham, "Millirio: Ego-motion estimation with low-cost millimetre-wave radar," IEEE Sensors Journal, vol. 21, no. 3, pp. 3314-3323, 2020.

[13] S. H. Cen and P. Newman, "Precise ego-motion estimation with millimeter-wave radar under diverse and challenging conditions," in 2018 IEEE International Conference on Robotics and Automation (ICRA), pp. 6045-6052, IEEE, 2018.

[14] G. Park, S. B. Choi, D. Hyun, and J. Lee, "Integrated observer approach using in-vehicle sensors and gps for vehicle state estimation," Mechatronics, vol. 50, pp. 134-147, 2018.

[15] D. M. Bevly, J. C. Gerdes, C. Wilson, and G. Zhang, "The use of gps based velocity measurements for improved vehicle state estimation," in Proceedings of the 2000 American Control Conference. ACC (IEEE Cat. No. 00CH36334), vol. 4, pp. 2538-2542, IEEE, 2000.

[16] V. Rodrigo Marco, J. Kalkkuhl, J. Raisch, and T. Seel, "A novel imu extrinsic calibration method for mass production land vehicles," Sensors, vol. 21 , no. 1 , p. 7,2021
[17] R. Kang, L. Xiong, M. Xu, J. Zhao, and P. Zhang, "Vins-vehicle: A tightly-coupled vehicle dynamics extension to visual-inertial state estimator," in 2019 IEEE Intelligent Transportation Systems Conference (ITSC), pp. 3593-3600, IEEE, 2019.

[18] H. Sasaki and T. Nishimaki, "A side-slip angle estimation using neural network for a wheeled vehicle," SAE transactions, pp. 1026-1031, 2000.

[19] Y. Liang, S. Müller, D. Rolle, D. Ganesch, and I. Schaffer, "Vehicle sideslip angle estimation with deep neural network and sensor data fusion," in 10th International Munich Chassis Symposium 2019, pp. 159-178, Springer, 2020.

[20] J. Lee, D. Hyun, K. Han, and S. Choi, "Real-time longitudinal location estimation of vehicle center of gravity," International Journal of Automotive Technology, vol. 19, no. 4, pp. 651-658, 2018.

[21] S. L. Miller, B. Youngberg, A. Millie, P. Schweizer, and J. C. Gerdes, "Calculating longitudinal wheel slip and tire parameters using gps velocity," in Proceedings of the 2001 American Control Conference.(Cat. No. 01CH37148), vol. 3, pp. 1800-1805, IEEE, 2001.

[22] D. M. Bevly, R. Sheridan, and J. C. Gerdes, "Integrating ins sensors with gps velocity measurements for continuous estimation of vehicle sideslip and tire cornering stiffness," in Proceedings of the 2001 American Control Conference.(Cat. No. 01CH37148), vol. 1, pp. 25-30, IEEE, 2001.

[23] G. Baffet, A. Charara, and D. Lechner, "Estimation of vehicle sideslip, tire force and wheel cornering stiffness," Control Engineering Practice, vol. 17, no. 11, pp. 1255-1264, 2009.

[24] G. Reina, M. Paiano, and J.-L. Blanco-Claraco, "Vehicle parameter estimation using a model-based estimator," Mechanical Systems and Signal Processing, vol. 87, pp. 227-241, 2017.

[25] K. Han, M. Choi, and S. B. Choi, "Estimation of the tire cornering stiffness as a road surface classification indicator using understeering characteristics," IEEE Transactions on Vehicular Technology, vol. 67, no. 8, pp. 6851-6860, 2018.

[26] M. C. Best, A. P. Newton, and S. Tuplin, "The identifying extended kalman filter: parametric system identification of a vehicle handling model," Proceedings of the Institution of Mechanical Engineers, Part K: Journal of Multi-body Dynamics, vol. 221, no. 1, pp. 87-98, 2007.

[27] C. Lundquist and T. B. Schön, "Recursive identification of cornering stiffness parameters for an enhanced single track model," IFAC Proceedings Volumes, vol. 42, no. 10, pp. 1726-1731, 2009.

[28] D. Wesemeier and R. Isermann, "Identification of vehicle parameters using stationary driving maneuvers," Control engineering practice, vol. 17, no. 12, pp. 1426-1431, 2009.

[29] Y. Wu, M. Wu, X. Hu, and D. Hu, "Self-calibration for land navigation using inertial sensors and odometer: Observability analysis," in AIAA Guidance, Navigation, and Control Conference, p. 5970, 2009.

[30] Q. Chen, Q. Zhang, and X. Niu, "Estimate the pitch and heading mounting angles of the imu for land vehicular gnss/ins integrated system," IEEE transactions on intelligent transportation systems, 2020.

[31] T. Wenzel, K. Burnham, M. Blundell, and R. Williams, "Kalman filter as a virtual sensor: applied to automotive stability systems," Transactions of the Institute of Measurement and Control, vol. 29, no. 2, pp. 95-115, 2007.

[32] T. A. Wenzel, K. Burnham, M. Blundell, and R. Williams, "Dual extended kalman filter for vehicle state and parameter estimation," Vehicle system dynamics, vol. 44, no. 2, pp. 153-171, 2006.

[33] S. J. Julier, J. K. Uhlmann, and H. F. Durrant-Whyte, "A new approach for filtering nonlinear systems," in Proceedings of 1995 American Control Conference-ACC'95, vol. 3, pp. 1628-1632, IEEE, 1995.

[34] S. J. Julier and J. K. Uhlmann, "New extension of the kalman filter to nonlinear systems," in Signal processing, sensor fusion, and target recognition VI, vol. 3068, pp. 182-193, International Society for Optics and Photonics, 1997.

[35] E. Tunali and T.-J. Tarn, "New results for identifiability of nonlinear systems," IEEE Transactions on Automatic Control, vol. 32, no. 2, pp. 146-154, 1987.

[36] A. F. Villaverde, N. Tsiantis, and J. R. Banga, "Full observability and estimation of unknown inputs, states and parameters of nonlinear biological models," Journal of the Royal Society Interface, vol. 16, no. 156, p. 20190043, 2019.

[37] J. Karlsson, M. Anguelova, and M. Jirstrand, "An efficient method for structural identifiability analysis of large dynamic systems," IFAC proceedings volumes, vol. 45, no. 16, pp. 941-946, 2012.

[38] S. G. Klauer, T. A. Dingus, V. L. Neale, J. D. Sudweeks, and D. J. Ramsey, "Comparing real-world behaviors of drivers with high versus low rates of crashes and near crashes," tech. rep., 2009.

[39] U. Kiencke and L. Nielsen, "Automotive control systems: for engine, driveline, and vehicle," 2000 
[40] H. B. Pacejka and R. S. Sharp, "Shear force development by pneumatic tyres in steady state conditions: a review of modelling aspects," Vehicle system dynamics, vol. 20, no. 3-4, pp. 121-175, 1991.

[41] M. K. Salaani, "Analytical tire forces and moments model with validated data," SAE Transactions, pp. 735-763, 2007.

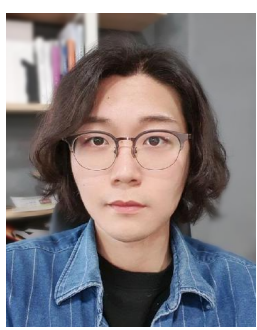

Yoonjin Hwang received the B.S. degree in physics and mechanical engineering from Sogang University, Seoul, South Korea, in 2013 and the M.S. degree in mechanical engineering from Korea Advanced Institute of Science and Technology(KAIST), Daejeon, South Korea in 2015 , where he is currently working toward the Ph.D. degree.

From 2016, He is co-founder and chief technical officer of 3 Secondz Inc., which is a company supplying driving data acquisition and automated report system. His research interests include inertial navigation, pose estimation, sensor fusion for ADAS, vehicle dynamics, and autonomous vehicle operating system.

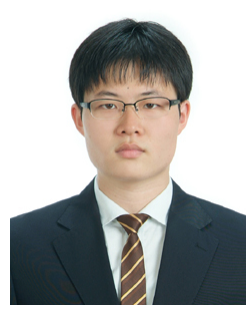

Yongseop Jeong received the B.S. degree in Electrical and Computer Engineering from Sungkyunkwan University, Seoul, South Korea, in 2011 and the M.S. degree in Robotics Program from Korea Advanced Institute of Science and Technology(KAIST), Daejeon, South Korea in 2013, where he is currently working toward the Ph.D. degree.

His research interests include sensor fusion, system integration for perception of autonomous vehicles.

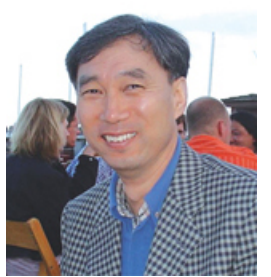

In So Kweon received the B.S. and the M.S. degrees in Mechanical Design and Production Engineering from Seoul National University, Korea, in 1981 and 1983, respectively, and the Ph.D. degree in Robotics from the Robotics Institute at Carnegie Mellon University in 1990.

He worked for Toshiba R\&D Center, Japan, and joined KAIST in 1992. He is KEPCO endowed chair professor of electrical engineering $(\mathrm{EE})$ and the director for the National Core Research Center - P3 DigiCar Center at KAIST.

Professor Kweon's research interests include computer vision and robotics. He has co-authored several books, including "Metric Invariants for Camera Calibration," and more than 300 technical papers. He served as a Founding Associate-Editor-in-Chief for "International Journal of Computer Vision and Applications", and has been an Editorial Board Member for "International Journal of Computer Vision" since 2005.

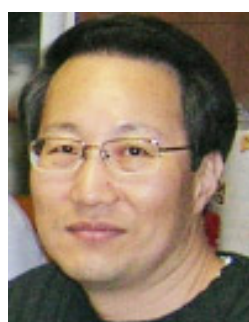

Seibum B. Choi (M'09) received the B.S. degree in mechanical engineering from Seoul $\mathrm{Na}$ tional University, Seoul, Korea in 1985, the M.S. degree in mechanical engineering from the Korea Advanced Institute of Science and Technology(KAIST), Daejeon, South Korea, in 1987, and the Ph.D. degree in control from the University of California, Berkeley, CA, USA, in 1993.

From 1993 to 1997, he was involved in the development of automated vehicle control systems at the Institute of Transportation Studies, University of California. In 2006, he was with TRW, Warren, MI, USA, where he was involved in the development of advanced vehicle control systems. Since 2006, he has been with the faculty of the Department of Mechanical Engineering, KAIST. His current research interets include fuel-saving technologies, vehicle dynamics and control, and active safety systems. 